PROCEEDINGS OF THE

AMERICAN MATHEMATICAL SOCIETY

Volume 140, Number 6, June 2012, Pages 2179-2181

S 0002-9939(2011)11173-0

Article electronically published on September 30, 2011

\title{
A NECESSARY AND SUFFICIENT CONDITION FOR RICCI SHRINKERS TO HAVE POSITIVE AVR
}

\author{
BENNETT CHOW, PENG LU, AND BO YANG
}

(Communicated by Jianguo Cao)

\begin{abstract}
In this short paper we observe that a recent result of C.-W. Chen meshes well with earlier work of H.-D. Cao and D.-T. Zhou, O. Munteanu, J. Carrillo and L. Ni, and S.-J. Zhang. We give a necessary and sufficient condition for complete noncompact shrinking gradient Ricci solitons to have positive asymptotic volume ratio.
\end{abstract}

Let $\left(\mathcal{M}^{n}, g, f\right)$ denote a complete shrinking gradient Ricci soliton (shrinker for short) with $R_{i j}+\nabla_{i} \nabla_{j} f-\frac{1}{2} g_{i j}=0$. Throughout we shall assume that $f$ is the normalized potential function in the sense that $R+|\nabla f|^{2}-f=0$ holds on $\mathcal{M}$.

It was proved by B.-L. Chen [3] that complete ancient solutions to the Ricci flow, and in particular shrinkers, must have nonnegative scalar curvature. As a consequence, the potential function $f$ satisfies the estimate:

$$
0 \leq f(x) \leq\left(\frac{1}{2} r(x)+f(O)^{\frac{1}{2}}\right)^{2},
$$

where $r(x)$ denotes the distance function to a fixed point $O$ in $\mathcal{M}$. H.-D. Cao and Detang Zhou [1] proved that there exists a positive constant $C$ which depends on the dimension $n, \sup _{y \in B(O, 1)}|\nabla f|(y)$, and the minimum of the Ricci curvature $\mathrm{Rc}_{g}$ in the ball $B(O, 1)$ such that $f$ satisfies the lower estimate

$$
f(x) \geq \frac{1}{4}(r(x)-C)^{2}
$$

for $x \in \mathcal{M}-B(O, C)$ (see Fang, Man, and Zhang $[5$ for related estimates). In fact, carefully following the proof of [1] and integrating by parts yield:

$$
f(x) \geq \frac{1}{4}\left[\left(r(x)-4 n-2 f(O)^{\frac{1}{2}}+\frac{4}{3}\right)_{+}\right]^{2},
$$

where $c_{+} \doteqdot \max (c, 0)$. Recently Haslhofer and Müller [6] further observed that if the reference point $O$ is chosen to be a global minimum point of $f$ (its existence is ensured by (11) and (2)), then one obtains improved estimates with constants depending only on $n$ :

$$
\frac{1}{4}\left[(r(x)-5 n)_{+}\right]^{2} \leqslant f(x) \leqslant \frac{1}{4}(r(x)+\sqrt{2 n})^{2} .
$$

Received by the editors February 2, 2011.

2010 Mathematics Subject Classification. Primary 53Cxx.

(C)2011 American Mathematical Society Reverts to public domain 28 years from publication 
Define the functions

$$
\mathrm{V}: \mathbb{R} \rightarrow[0, \infty), \quad \mathrm{R}: \mathbb{R} \rightarrow[0, \infty)
$$

by

$$
\mathrm{V}(c) \doteqdot \int_{\{f<c\}} d \mu, \quad \mathrm{R}(c) \doteqdot \int_{\{f<c\}} R d \mu .
$$

In [1, the following ODE relating $\mathrm{V}(c)$ and $\mathrm{R}(c)$ was established:

$$
0 \leq \frac{n}{2} \mathrm{~V}(c)-\mathrm{R}(c)=c \mathrm{~V}^{\prime}(c)-\mathrm{R}^{\prime}(c) .
$$

Recall that the asymptotic volume ratio (AVR) of a complete noncompact Riemannian manifold $\left(\mathcal{N}^{n}, h\right)$ is defined by

$$
\operatorname{AVR}(h) \doteqdot \lim _{r \rightarrow \infty} \frac{\operatorname{Vol} B(p, r)}{\omega_{n} r^{n}}
$$

if the limit exists, where $B(p, r)$ denotes the geodesic ball in $\mathcal{N}$ with center $p$ and radius $r$ and where $\omega_{n}$ is the volume of the unit Euclidean $n$-ball. It is easy to check that the $\operatorname{AVR}(h)$ is independent of the choice of $p$. Moreover, if $h$ has nonnegative Ricci curvature, then this limit exists by the Bishop-Gromov volume comparison theorem.

H.-D. Cao and Detang Zhou [1] proved the following using (5) and aided by an observation of Munteanu [7] (see [6] for an improvement).

Theorem 1. Any complete noncompact shrinking gradient Ricci soliton must have at most Euclidean volume growth; i.e., $\lim _{\sup _{r \rightarrow \infty}} \frac{\operatorname{Vol} B(O, r)}{\omega_{n} r^{n}}$ is finite.

Note that an earlier result by Carrillo and $\mathrm{Ni} 2$ states that any nonflat shrinker with nonnegative Ricci curvature must have zero AVR. Based on Cao and Zhou's work, Zhang [8] proved a sharp upper bound on the volume growth of shrinkers under the assumption that $R \geq \delta$ for some constant $\delta>0$. More recently, C.-W. Chen [4] proved that the AVR of a shrinker is bounded from below by some $c>0$ if the average scalar curvature satisfies $\frac{1}{\operatorname{Vol} B(O, r)} \int_{B(O, r)} R d \mu \leq r^{\alpha}$, where $\alpha$ is a negative constant (see also [1 for a similar result in the case where $\alpha=0$ ).

We observe that the results in 2], 1, 7], 8, and [4] lead to a necessary and sufficient condition for noncompact shrinkers to have positive AVR.

Theorem 2. Let $\left(\mathcal{M}^{n}, g, f\right)$ be a complete noncompact shrinking gradient Ricci soliton. Then $\operatorname{AVR}(g)$ exists and is finite (by [6], it is bounded by a constant depending only on $n)$. Moreover, $\operatorname{AVR}(g)>0$ if and only if $\int_{n+2}^{\infty} \frac{\mathrm{R}(c)}{c \mathrm{~V}(c)} d c<\infty$.

Proof. Let $\mathrm{P}(c) \doteqdot \frac{\mathrm{V}(c)}{c^{\frac{n}{2}}}-\frac{\mathrm{R}(c)}{c^{\frac{n}{2}+1}}$ and $\mathrm{N}(c) \doteqdot \frac{\mathrm{R}(c)}{c \mathrm{~V}(c)}$. Note that $\frac{\mathrm{R}(c)}{\mathrm{V}(c)}$ is the average scalar curvature over the set $\{f<c\}$. The ODE (15) implies that

$$
\mathrm{P}^{\prime}(c)=-\left(1-\frac{n+2}{2 c}\right) \frac{\mathrm{R}(c)}{c^{\frac{n}{2}+1}}=-\frac{\left(1-\frac{n+2}{2 c}\right) \mathrm{N}(c)}{1-\mathrm{N}(c)} \mathrm{P}(c) .
$$

Since $0 \leq \mathrm{R}(c) \leq \frac{n}{2} \mathrm{~V}(c)$ by (5), we have

$$
\left(1-\frac{n}{2 c}\right) \frac{\mathrm{V}(c)}{c^{\frac{n}{2}}} \leq \mathrm{P}(c) \leq \frac{\mathrm{V}(c)}{c^{\frac{n}{2}}} .
$$


Hence, by the bounds (11) and (2) for $f$,

$$
2^{n} \omega_{n} \operatorname{AVR}(g)=\lim _{c \rightarrow \infty} \frac{\mathrm{V}(c)}{c^{n / 2}}=\lim _{c \rightarrow \infty} \mathrm{P}(c),
$$

which exists by (7).

Integrating (7) yields

$$
\mathrm{P}(c)=\mathrm{P}(n+2) e^{-\int_{n+2}^{c} \frac{\left(1-\frac{n+2}{2 c}\right) \mathrm{N}(c)}{1-\mathrm{N}(c)} d c}
$$

for $c \geq n+2$. From $\frac{\mathrm{R}(c)}{\mathrm{V}(c)} \leq \frac{n}{2}$ it is easy to see that for any $c \in[n+2, \infty)$ we have

$$
\frac{1}{2} \int_{n+2}^{c} \mathrm{~N}(c) d c \leq \int_{n+2}^{c}\left(1-\frac{n+2}{2 c}\right) \frac{\mathrm{N}(c)}{1-\mathrm{N}(c)} d c \leq 2 \int_{n+2}^{c} \mathrm{~N}(c) d c .
$$

If $\int_{n+2}^{\infty} \mathrm{N}(c) d c=\infty$, then by (9) we have $\operatorname{AVR}(g)=\frac{1}{2^{n} \omega_{n}} \lim _{c \rightarrow \infty} \mathrm{P}(c)=0$.

If $\int_{n+2}^{\infty} \mathrm{N}(c) d c<\infty$, then by (9) and (10), we have

$$
\mathrm{P}(c) \geq \mathrm{P}(n+2) e^{-2 \int_{n+2}^{\infty} \mathrm{N}(c) d c}>0 .
$$

Hence $\operatorname{AVR}(g)>0$.

\section{ACKNOWLEDGMENT}

We would like to thank Lei Ni for his interest and encouragement.

\section{REFERENCES}

[1] Cao, Huai-Dong, Zhou, Detang, On complete gradient shrinking Ricci solitons. Journal of Differential Geometry 85 (2010), 175-185. MR2732975

[2] Carrillo, José A., Ni, Lei, Sharp logarithmic Sobolev inequalities on gradient solitons and applications. Communications in Analysis and Geometry 17 (2009), 721-753.

[3] Chen, Bing-Long, Strong uniqueness of the Ricci flow. Journal of Differential Geometry 82 (2009), 363-382. MR2520796 (2010h:53095)

[4] Chen, Chih-Wei, On the injectivity radius and tangent cones at infinity of gradient Ricci solitons. arXiv:1012.1217.

[5] Fang, Fu-Quan, Man, Jian-Wen, Zhang, Zhen-Lei, Complete gradient shrinking Ricci solitons have finite topological type. C. R. Math. Acad. Sci. Paris 346 (2008), no. 11-12, 653-656. MR2423272 (2009e:53043)

[6] Haslhofer, Robert, Müller, Reto, A compactness theorem for complete Ricci shrinkers. arXiv:1005.3255v2.

[7] Munteanu, Ovidiu, The volume growth of complete gradient shrinking Ricci solitons. arXiv:0904.0798

[8] Zhang, Shijin. On a sharp volume estimate for gradient Ricci solitons with scalar curvature bounded below. arXiv:0909.0716, Acta Mathematica Sinica 27, no. 5 (2011), 871-882.

Department of Mathematics, University of California San Diego, La Jolla, CaliforNIA 92093

E-mail address: benchow@math.ucsd.edu

Department of Mathematics, University of Oregon, Eugene, Oregon 97403

E-mail address: penglu@uoregon.edu

Department of Mathematics, University of California San Diego, La Jolla, CaliforNIA 92093

E-mail address: b5yang@math.ucsd.edu 